\title{
Lernziele: «nice to have» oder «need to have»?
}

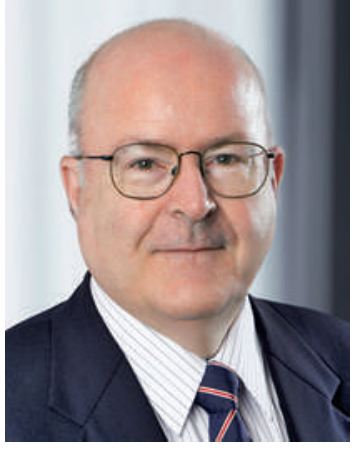

Wo es um Schule, Studium und Weiterbildung geht, flackern immer wieder Diskussionen zu den Lernzielkatalogen auf. Es geht dabei meist um zwei Aspekte:

- Wie sollen Lernziele formuliert werden? Müssen sie das Wissen und einzelne Fertigkeiten detailliert beschreiben oder sollten sie mehr in Richtung von breiter definierten Kompetenzen gehen?

- Wie vollständig hat ein Lernzielkatalog zu sein? Geht es um eine Auswahl von Wichtigem oder darf möglichst kein einzelnes Lernziel vergessen gehen?

Ein eindrückliches nicht-medizinisches Beispiel bieten im Moment die Auseinandersetzungen um den «Lehrplan 21» für die Volksschule, wo sich das «alte» didaktische Konzept einer konkreten Lernstoffsammlung einem «fortschrittlichen» Konzept mit primärer Kompetenzorientierung gegenüber sieht. Unbestritten ist dieser Wandel nicht. In einer Stellungnahme versucht denn auch die pädagogische Hochschule Zürich Bedenken zu entkräften, wonach «es später nur noch globalisierte und pauschalisierte Grundkompetenzen gebe statt des verankerten, für das Berufsleben wichtigen Fachwissens».

\section{We expect too much of the student and we try to teach him too much (W. Osler).}

Auch wo es um ärztliche Bildung geht, sind wir heute mit der wichtigen Frage konfrontiert, wie Lernziele in Zukunft zu formulieren sind und wie umfassend Lernzielkataloge sein müssen:

- Soeben beginnt eine Überarbeitung des Schweizerischen Lernzielkatalogs für das Medizinstudium (SCLO). Eine erste Weichenstellung wird dabei sein, inwieweit in Ergänzung einer Liste essentieller Einzelziele auch Kompetenzen mit integrierten Kenntnissen und Fähigkeiten eingebaut werden sollen. Zudem stellt sich die Frage, wie mit der schon überlangen Liste einzelner Ziele zu verfahren ist, in der viele Spezialfächer sich immer noch nicht genügend abgebildet sehen.

- Ab Mitte 2015 können Anträge für einen Facharzttitel nur noch in Form eines e-Logbuches eingereicht werden. Bei dessen Erarbeitung mussten die Projektverantwortlichen im Schweizerischen Institut für ärztliche Weiter- und Fortbildung SIWF erkennen, dass die Formulierung der Lernziele in den einzelnen Weiterbildungsprogrammen auf keiner gemeinsamen Systematik aufbaut. Die einen zählen Eingriffe, die anderen vergeben Punkte, andere sprechen von «erfüllt», aber alle gehen sehr weit in Richtung Details und Vollständigkeit. In Zukunft müssen auch im Hinblick auf die elektronische Verarbeitung und die internationale Diplomanerkennung die Lernzielkataloge bis zu einem gewissen Grad einheitlich strukturiert sein

\section{Ärztliche Bildung muss zunehmend auch fächerübergreifende Lernziele berücksichtigen.}

- Die schweizerischen Weiterbildungsgänge werden alle sieben Jahre vom Bund akkreditiert, das nächste Mal im Jahr 2018. Es gilt, schon in den nächsten zwei Jahren in den Fachgesellschaften die Voraussetzungen hierfür zu schaffen. Dazu gehören Überlegungen, welche Kompetenzen im Laufe der Weiterbildung erworben und wie sie definiert werden müssen - immer aufbauend auf der Grundausbildung im Studium. Dabei ist dem Wandel der Methoden und Berufsbilder Rechnung zu tragen.

- Eine Umfrage der ETH bei Ärztinnen und Ärzten, welche die Weiterbildung vor einigen Jahren abgeschlossen haben, ergab quer durch alle Fachdisziplinen ein gefühltes Defizit in den Bereichen von Kommunikation, ökonomischen Grundlagen, Fehleranalyse und Festlegung der Arbeitsfähigkeit. Hier geht es um fächerübergreifende, allgemeine Lernziele, die in den Kompetenzrucksack jeder Ärztin und jedes Arztes gehören. Deren Formulierung ist hier wohl weniger schwierig als die Umsetzung im Alltag.

Im Bericht über die Plenarversammlung des SIWF und das Referat von Herrn Professor P. Berberat aus München (S. 1843 in dieser Ausgabe der Schweizerischen Ärztezeitung) werden einzelne Aspekte dieser Problematik zusammenfassend dargestellt. Gemeinsam mit den medizinischen Fakultäten und den Fachgesellschaften ist das SIWF gefordert, die Lernziele für die Zukunft sinnvoll auszuwählen, zu formulieren und auch Methoden für die nötige Evaluation zu validieren.

Dr. med. Werner Bauer, Präsident des Schweizerischen Instituts für ärztliche Weiter- und Fortbildung SIWF 